\title{
Scanned-cantilever atomic force microscope
}

\author{
David R. Baselt and John D. Baldeschwieler \\ Division of Chemistry, California Institute of Technology 127-72, Pasadena, California 91125
}

(Received 28 September 1992; accepted for publication 11 December 1992)

We have developed a $3.6 \mu \mathrm{m}$ scan range atomic force microscope that scans the cantilever instead of the sample, while the optical-lever detection apparatus remains stationary. The design permits simpler, more adaptable sample mounting, and generally improves ease of use. Software workarounds alleviate the minor effects of spurious signal variations that arise as a result of scanning the cantilever. The performance of the microscope matches that of scanned-sample instruments.

\section{INTRODUCTION}

The atomic force microscope (AFM) ${ }^{1}$ measures topography (potentially at atomic resolution) by scanning a small tip, attached to a cantilever spring, over a sample. The deflection of the cantilever indicates the sample height at the tip position. The most popular technique used to measure the deflection is the "optical lever,",2,3 in which a position-sensitive photodiode measures the angular deflection of a laser beam refiected from the cantilever. A feedback loop monitors the cantilever deflection and keeps itand thus the force exerted on the sample-constant.

All AFMs described in the literature to date ${ }^{1-5}$ scan the sample in order to keep the cantilever stationary with respect to the detection apparatus. This configuration restricts the sample mounting area to the end of the scanner, a region at most $0.5 \mathrm{in}$. in diameter. Massive samples degrade instrument performance by lowering the natural resonant frequency of the scanner. In addition, access to the sample for translation or replacement can be somewhat inconvenient.

Below, we describe a scanned-cantilever AFM that alleviates these problems. The instrument is easier to work with and more flexible than scanned-sample AFMs. Although other scanned-cantilever AFMs (not described in the literature) have been developed independently of ours, they are all large scan range instruments that move the entire detection apparatus-in the case of optical lever instruments a unit containing the laser, cantilever, and detector scans over the sample. In contrast, our instrument scans only the cantilever, resulting in superior performance at small scan ranges.

\section{DESIGN}

Our implementation of the scanned-cantilever AFM (Fig. 1) positions the sample face-down at the top of the instrument to allow maximum accessibility. The cantile$\operatorname{ver}^{6}$ is mounted on a $3.6 \mu \mathrm{m}$ range, $0.5 \mathrm{in}$. long $\times 0.5 \mathrm{in}$. diam $\times 0.020$ in. thick tube of EBL No. 2 piezoceramic. ${ }^{7} A$ focused diode laser, ${ }^{8}$ mounted on a tiltable platform at the bottom of the instrument, emits a beam that shines up through the scanner to reflect off the cantilever. The laser beam then reflects off a rotatable mirror assembly into a four-segment position-sensitive photodetector. ${ }^{9}$ Two 80 tpi thumbscrews and a motorized screw ${ }^{10}$ serve to approach the tip and sample.

The sample mount accommodates a standard glass microscope slide. Since many of our samples are biomolecules deposited on glass, it is usually possible to view the tip and sample (through the slide) with an optical microscope while scanning.

The inverted-sample configuration of our AFM complicates liquid flow cell design (note that the scannedcantilever design does not require an inverted-sample configuration). After trying a number of flow cell designs we have settled on one that uses two rubber $O$ rings and a latex glove rubber seal as illustrated in Fig. 2. The rubber seal has a hole in its center to admit laser light, and has sufficient elasticity to allow a tip-sample approach.

The scanned-cantilever design has the considerable advantage that all active parts of the instrument-scanner, optical lever, and tip-sample approach mechanism-are contained in the "base." The "head," which holds the sample, has no wires or other attachments (unless the flow cell is in use) and thus can be removed and handled with ease.

\section{PERFORMANCE}

Scanning the cantilever while the optical-lever detection apparatus remains stationary could potentially give rise to spurious variations of the cantilever deflection signal. In turn, this would cause variations in the force exerted on the sample. We address here the theoretical effects of three possible sources of spurious signal variations.

As illustrated in Fig. 3(a), the cantilever scans in $X$ and $Y$ (the directions parallel to the sample) while the beam reflected from it remains stationary; therefore, imperfections of the cantilever surface could cause fluctuations in the reflected laser beam as a scan progresses. These "imperfections" include the edge of the cantilever (that is, the cantilever could move out from under the beam), particles of dust, small surface imperfections (such as scratches), and overall warping of the cantilever. Given that the cantilever width is at least $25 \mu \mathrm{m}$ and the beam diameter is $10 \mu \mathrm{m}$, the maximum scan size of $3.6 \mu \mathrm{m}$ is small enough that we do not have to worry about the cantilever moving out from under the beam. The fact that the beam diameter exceeds the scan size also means that any spurious signal variations due to cantilever irregulari- 


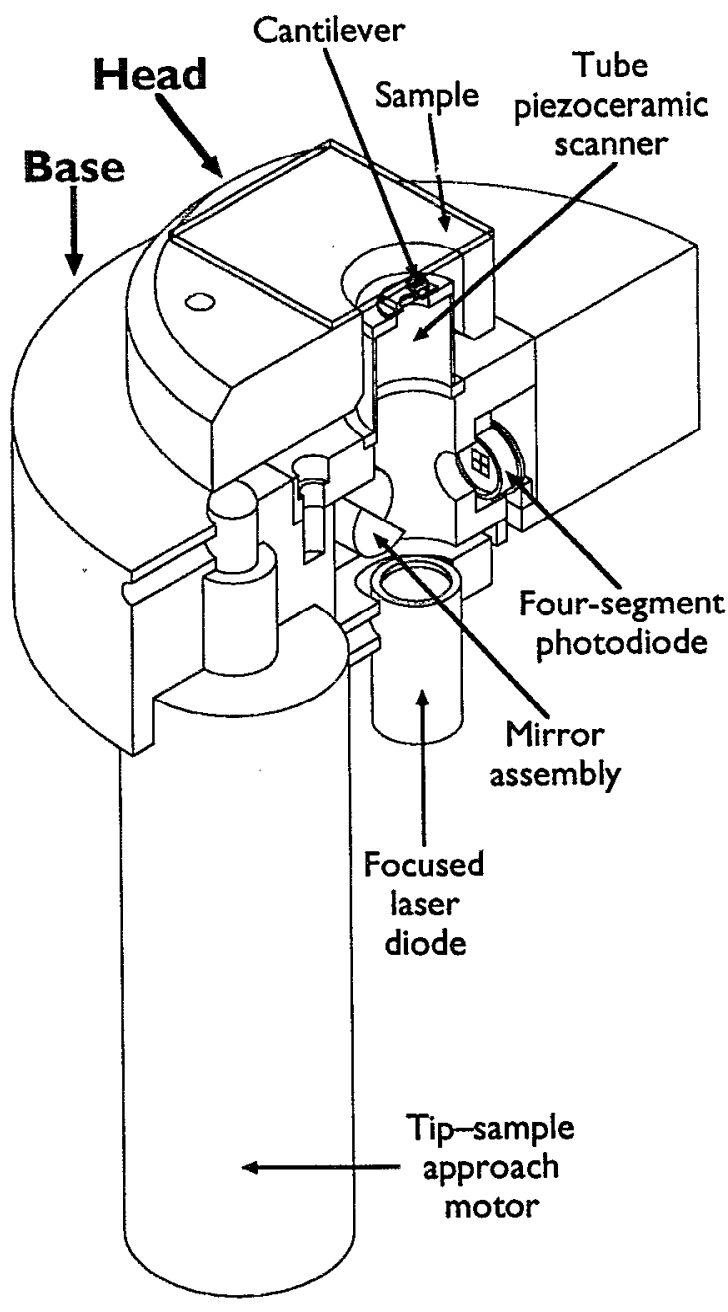

FIG. 1. Cross section of the scanned-cantilever, optical-lever AFM. The stage measures 3 in. in diameter. The sample, a glass microscope slide, is held face-down on the head with spring clips or a clamp (not shown).

ties should have a first-order effect that can be removed with a first-order background subtraction, as described below.

Figure $3(\mathrm{~b})$ diagrams the tilting of the scanner that accompanies $X Y$ motion. Since the detection apparatus is

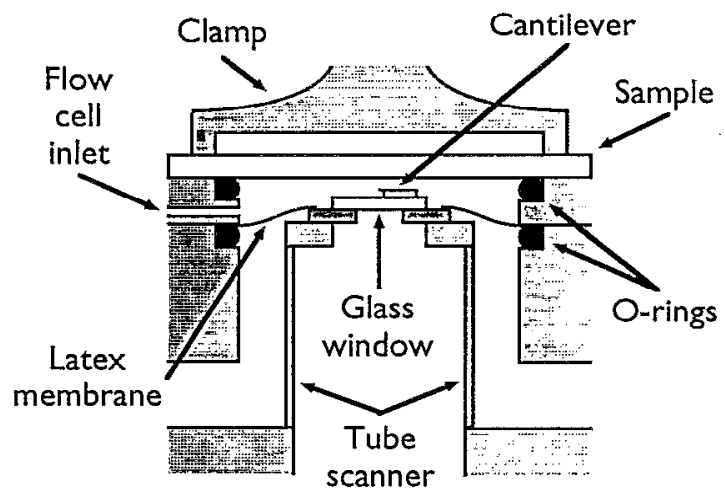

FIG. 2. Liquid flow cell for the inverted-sample configuration. A membrane of latex rubber seals of the liquid cell while providing the flexibility needed to adjust the sample height. A clamp holds the sample firmly against a rubber $O$ ring. a $\Delta X=3.6 \mu \mathrm{m}$
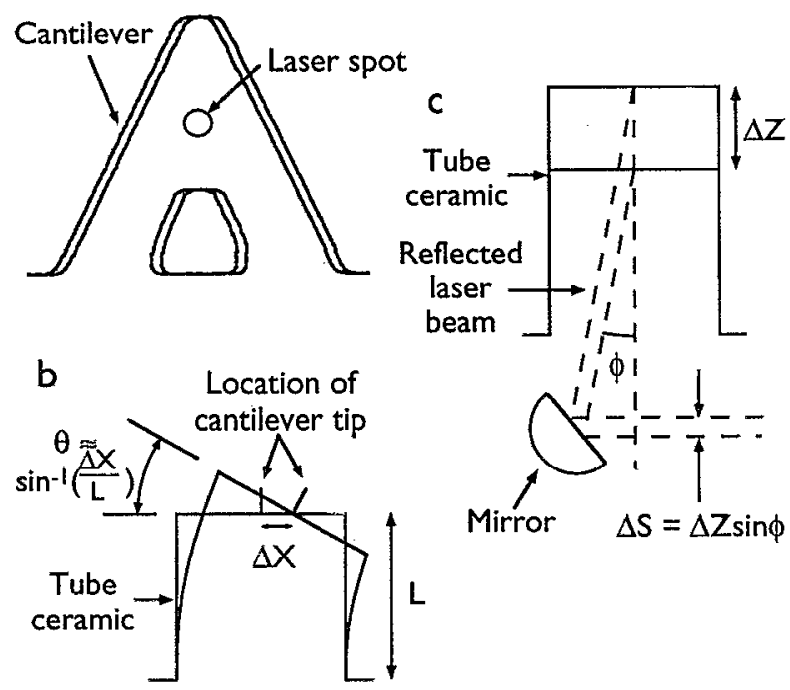

FIG. 3. Sources of spurious cantilever deflection signals. (a) Cantilever movement in $X$ and $Y$ with respect to the laser beam. The cantilever, traced from a photomicrograph of a Park Scientific cantilever, is shown at the extremes of the instrument scan range. The focused laser spot, drawn to scale, measures $10 \mu \mathrm{m}$ in diameter. (b) Scanner tilt with $X Y$ displacement. The tube ceramic length $L$ is 0.5 in. in our system. (c) $Z$ displacement of cantilever. The angle $\phi$ between the incident and reflected laser beams is about $10^{\circ}$ in our system.

unable to distinguish scanner tilt from cantilever deflection, a spurious signal variation results. Over a $1 \mu \mathrm{m}$ scan, the scanner tilts enough to produce a $40 \AA \AA$ signal variation. However, curvature of this magnitude is common even in scanned-sample AFMs, and thus we believe it is acceptable.

Finally, as illustrated in Fig. 3(c), the laser beam reflected from the cantilever is displaced as the cantilever moves in $Z$ (up and down). However, the effect is very small, $1 \AA$ of signal variation per $\mu \mathrm{m}$ of $Z$ displacement.

In practice, we often observe significant spurious variations in cantilever deflection while scanning, usually due either to improper alignment of the laser to the cantilever, poor focusing of the laser, or warping of the cantilever. Since we cannot easily correct it, the latter constitutes our most serious concern. By noting the location of the reflected laser beam in our AFM stage, we can estimate to about $1^{\circ}$ how much our cantilevers are warped. The commercial cantilevers we are currently using ${ }^{6}$ are generally warped $3^{\circ}-5^{\circ}$; that is, the end of the cantilever is raised up at this angle.

In theory a cantilever warped by $5^{\circ}$ should cause a spurious signal variation of $520 \AA / \mu \mathrm{m}$ of scan range in $Y$ (parallel with the long axis of the cantilever). The warp should not have any effect when moving the cantilever in the $X$ or $Z$ directions. In practice, the spurious signal variation in our instrument is close to this value: typically 600 $\AA / \mu \mathrm{m}$ of $Y$ displacement and $30 \AA / \mu \mathrm{m}$ of $X$ displacement over a $0.75 \mu \mathrm{m}$ displacement. The signal variation with $Z$ displacement is unmeasurable. For comparison, on a commercial scanned-sample instrument ${ }^{11}$ we measured signal 


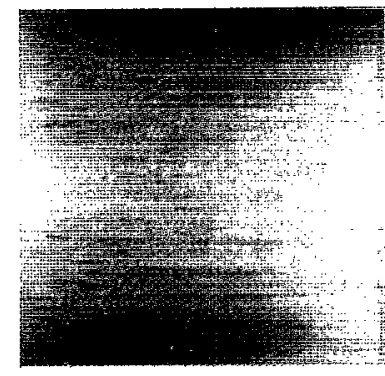

- (a) Leveled only

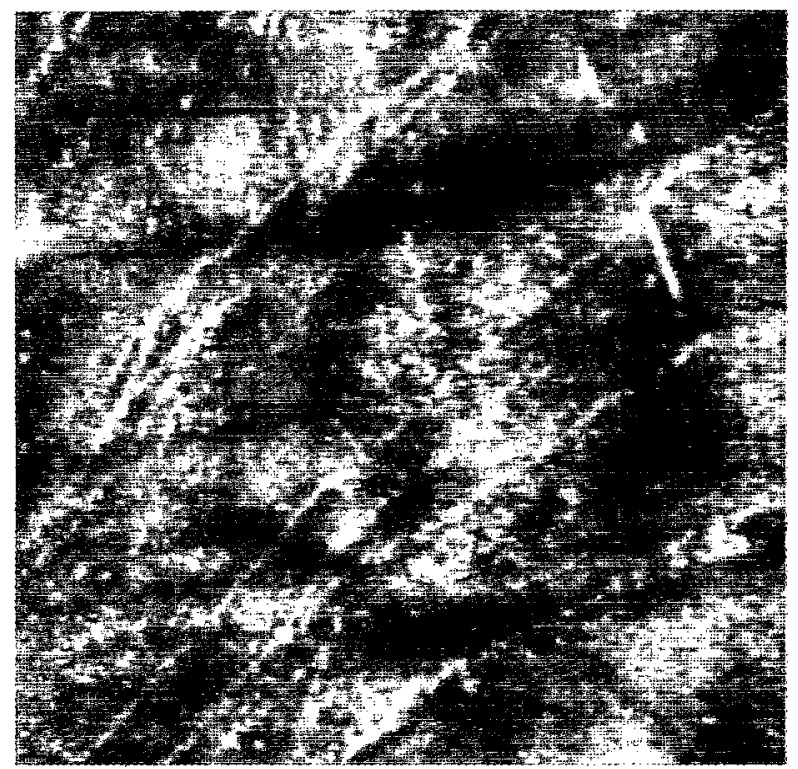

FIG. 4. A $1.6 \times 1.6 \mu \mathrm{m}$ topographic image of float-polished natural quartz. (a) Raw data after best-fit plane subtraction. The brightest and darkest areas differ by $140 \AA$. (b) After curvature removal by best-fit line subtraction from each horizontal and vertical line. The brightest and darkest areas differ by $14 \AA$. Wide, shallow polishing marks are visible, as are sharper grain defects. These features are apparently real, since similar features do not appear in images of atomically flat mica at the same magnification.

variations of $34 \AA / \mu \mathrm{m}$ in $Y, 7 \AA / \mu \mathrm{m}$ in $X$, and $330 \AA / \mu \mathrm{m}$ in $Z$ (possibly due to stray light reflecting from a metal backing on the sample). All of these measurements were taken with a glass microscope slide as the sample.

We have implemented a simple backgroundsubtraction algorithm that largely counters the effect of spurious signal variations. Before approaching the tip and sample, our instrument-control software measures the deflection signal as a function of position and calculates a background, which is subsequently subtracted while scanning. This background subtraction reduces fluctuations in force that occur as a result of spurious signal variations. The entire background measurement procedure takes only a second and does not detract from operation of the instrument. In fact, as noted above, we have observed spurious signal variations even in scanned-sample instruments, and thus recommend the background-subtraction procedure for all optical-lever AFMs. Although we only calculate a first-order background, higher-order subtraction is certainly possible.

Over the past year we have verified results from the scanned-cantilever AFM by repeating experiments per-

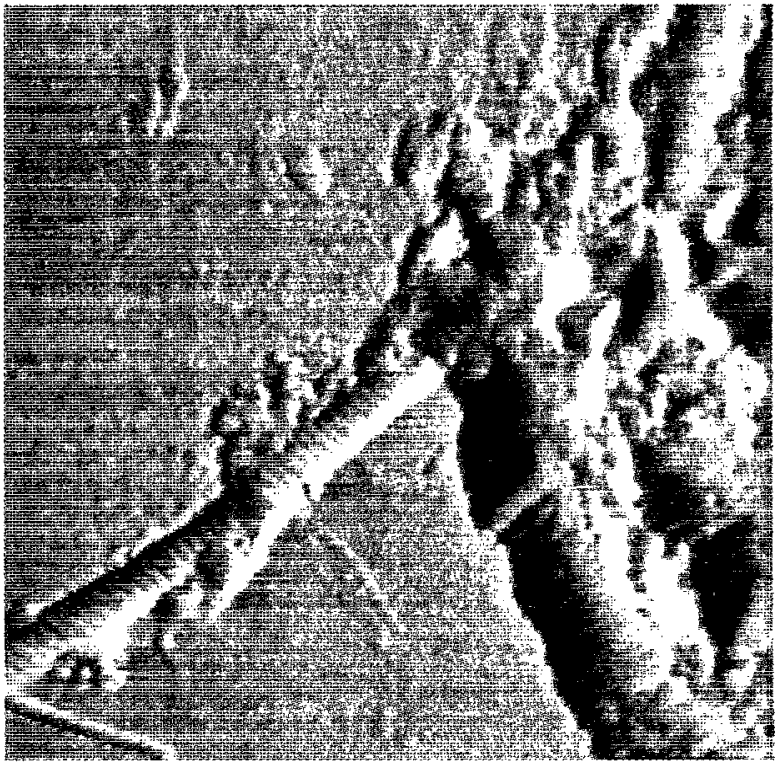

FIG. 5. A $2.4 \times 2.4 \mu \mathrm{m}$ normal deflection image of native rat tail collagen fibrils on glass. The characteristic $650 \AA$ period is clearly visible. Raw data, corrected for $X$ hysteresis.

formed on it with a commercial scanned-sample instrument. ${ }^{11}$ Other than the above mentioned signal variations, which are easily identified and removed, we have found no artifacts unique to the scanned-cantilever design.

In Fig. 4 we present an image of float-polished quartz, ${ }^{12}$ a very flat sample useful for judging the effects of image curvature. The raw data [Fig. 4(a)] shows curvature of about $140 \AA$ over a $1.6 \mu \mathrm{m}$ scan range, typical for our scanned-cantilever AFM (note that our commercial scanned-sample instrument often produces curvature equal to or greater than this). Given that our scanner produces scans theoretically planar to $0.4 \AA$, we conclude that background subtraction has left $88 \AA$ of spurious signal variation per $\mu \mathrm{m}$ of scan range. Since the cantilever used for this scan had a $0.3 \mathrm{~N} / \mathrm{m}$ spring constant the imaging force varied around the $10 \mathrm{nN}$ setpoint by $2.6 \mathrm{nN} / \mu \mathrm{m}$ of scan range.

A number of image processing techniques can remove such curvature. The relatively homogeneous image of quartz responds well to a flattening process in which a best-fit line is determined for and subtracted from each row and each column of pixels [Fig. 4(b)]. Images with prominent or unevenly distributed features respond better to high-pass filtering, best-fit paraboloid subtraction, or lineby-line median subtraction.

The data in Fig. 4 are also significant because the quartz samples were too large to image in a scannedsample AFM without cutting them into pieces, a process that could create artifactual defects or scratches.

We have also imaged collagen fibrils dried on glass (Fig. 5), an application for which the scanned-cantilever design is well suited. The tendancy of the fibrils to cluster together as seen in this image makes them difficult to locate by trial-and-error. Thus we have used an optical micro- 
scope as described above to locate regions with high fibril density and position them over the tip.

Other applications for which we have used this stage include lateral force measurements on the atomic scale and on graphite steps, ${ }^{13}$ DNA imaging, and elasticity measurements. The noise level of the instrument is $0.2 \AA \mathrm{rms}$ in the $0-10 \mathrm{kHz}$ bandwidth.

\section{ACKNOWLEDGMENTS}

We would like to thank Jean-Paul Revel for suggesting that we image collagen fibrils and for help preparing collagen samples. We would also like to thank Shubert Soares for the polished quartz samples and Topometrix, Inc. for the use of their electronics. This work was supported in part by a grant from the Ford Motor Company and an NSF predoctoral fellowship (D.B.).

${ }^{1}$ G. Binnig and C. F. Quate, Phys. Rev. Lett. 56, 930 (1986).

${ }^{2}$ G. Meyer and N. M. Amer, Appl. Phys. Lett. 53, 1045 (1988).

${ }^{3}$ S. Alexander, L. Hellemans, O. Marti, J. Schneir, V. Elings, and P. K. Hansma, J. Appl. Phys. 65, 164 (1989).

${ }^{4}$ C. A. J. Putman, K. O. van der Werf, Bart G. de Grooth, N. F. van
Hulst, F. B. Segerink, and J. Greve, Rev. Sci. Instrum. 63, 1914 (1992). ${ }^{5}$ For non-optical-lever designs, see for example $H$. Heinzelmann, P. Grütter, E. Meyer, H. Hidber, L. Rosenthaler, M. Ringger, and H.-J. Güntherodt, Surf. Sci. 189/190, 29 (1987) (tunneling); R. Erlandsson, G. M. McClelland, C. M. Mate, and S. Chiang, J. Vac. Sci. Technol. A 6, 266 (1988) (interferometry); and T. Göddenhenrich, H. Lemke, U. Hartmann, and C. Heiden, ibid. 8, 383 (1990) (capacitance).

${ }^{6}$ Microlevers or U1tralevers; Park Scientific Instruments, 1171 Borregas Ave., Sunnyvale, CA 94089. The amount of warp of Microlevers seems to vary from hatch to batch but generally seems to be less than $1^{\circ}$; our batch may be unusual.

${ }^{7}$ Staveley Sensors, EBL Company, 91 Prestige Park Circle, East Hartford, CT 06108. To reduce hysteresis and the possibility of accidental depoling, we have recently replaced the tube ceramic with a $1.8 \mu \mathrm{m}$ range EBL No. 1 tube of identical size. However, all data in this article were taken with the old EBL No. 2 scanner.

${ }^{8}$ Hoetron, Inc., 776 Palomar Ave., Sunnyvale, CA 94086.

${ }^{9}$ SPOT-4D, United Detector Technology, 12525 Chadron Ave., Hawthorne, CA 90250.

${ }^{10}$ Series 860 motorizer, Newport Corporation, 18235 Mt. Baldy Círcle, Fountain Valley, CA 92728.

${ }^{11}$ Topometrix TMX2000 $1 \mu \mathrm{m}$ AFM; Topometrix, Inc., 1505 Wyatt Drive, Santa Clara, CA 95054.

${ }^{12}$ S. F. Soares, D. R. Baselt, J. P. Black, K. C. Jungling, and W. K. Stowell (submitted).

${ }^{13}$ D. R. Baselt and J. D. Baldeschwieler, J. Vac. Sci. Technol. B 10, 2316 (1992). 